\title{
On the Geometry of Two-Dimensional Slices of Irregular Level Sets in Turbulent Flows
}

\author{
P. E. Dimotakis, H. J. Catrakis, A. W. Cook, J. M. Patton
}

This paper was prepared for submittal to the American Physical Society/Division of Fluid Dynamics

Philadelphia, PA

November 22 - 24, 1998

March 20, 1998

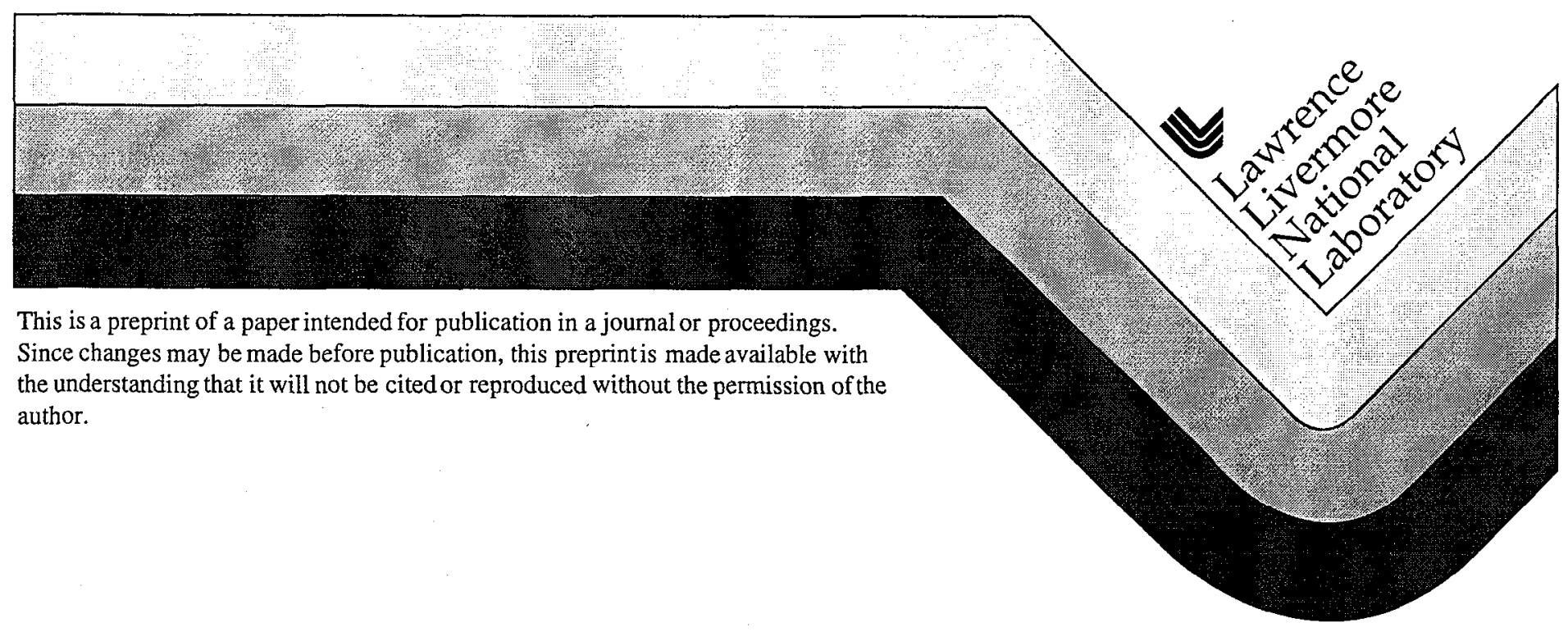




\section{DISCLAIMER}

This document was prepared as an account of work sponsored by an agency of the United States Government. Neither the United States Government nor the University of California nor any of their employees, makes any warranty, express or implied, or assumes any legal liability or responsibility for the accuracy, completeness, or usefulness of any information, apparatus, product, or process

disclosed, or represents that its use would not infringe privately owned rights. Reference herein to any specific commercial product, process, or service by trade name, trademark, manufacturer, or otherwise, does not necessarily constitute or imply its endorsement, recommendation, or favoring by the United States Government or the University of California. The views and opinions of authors expressed herein do not necessarily state or reflect those of the United States Government or the University of California, and shall not be used for advertising or product endorsement purposes. 


\title{
On the geometry of two-dimensional slices of irregular level sets in turbulent flows*
}

\author{
Paul E. Dimotakis, Haris J. Catrakis, Andrew W. Cook, ${ }^{* *}$ and James M. Patton ${ }^{\dagger}$ \\ Graduate Aeronautical Laboratories \\ California Institute of Technology \\ Pasadena, California 91125
}

\begin{abstract}
Isoscalar surfaces in turbulent flows are found to be more complex than (selfsimilar) fractals, in both the far field of liquid-phase turbulent jets and in a realization of Rayleigh-Taylor-instability flow. In particular, they exhibit a scaledependent coverage dimension, $D_{2}(\lambda)$, for 2-D slices of scalar level sets, that increases with scale, from unity, at small scales, to 2 , at large scales. For the jet flow and Reynolds numbers investigated, the isoscalar-surface geometry is both scalarthreshold- and Re-dependent; the level-set (coverage) length decreases with increasing $R e$, indicating enhanced mixing with increasing Reynolds number; and the size distribution of closed regions is well described by lognormal statistics at small scales. A similar $D_{2}(\lambda)$ behavior is found for level-set data of 3-D density-interface behavior in recent direct numerical-simulation studies of Rayleigh-Taylor-instability flow. A comparison of (spatial) spectral and isoscalar coverage statistics will be discussed.
\end{abstract}

\section{Introduction}

Following the introduction of Fourier spectra in the analysis of velocity correlations and the decomposition of turbulent kinetic-energy by G. I. Taylor (1938), descriptions of turbulent flow have largely relied on spectral measurement and analysis methods. Subsequent contributions introduced the notion of similarity in the description of small-scale turbulence (Kolmogorov 1941a,b,c; "K41") as discussed by G. K. Batchelor (1953) and J. O. Hinze (1975), with important refinements and extensions subsequently put forth (e.g., Monin \& Yaglom 1975, Frisch 1995).

* Presented at the $2^{\text {nd }}$ Monte Verita Colloquium on Fundamental Problematic Issues in Turbulence, 22-28 March 1988 (Ascona, Switzerland).

** Lawrence Livermore National Laboratories, Livermore, California 94550.

$\dagger$ Center for Advanced Computing Research, California Institute of Technology.

This work was performed under the auspices of the U.S. Department of Energy by Lawrence Livermore National Laboratory under contract No. 
The successes of these proposals, however, must be tempered by the host of turbulent-flow issues that cannot be addressed by correlation/spectral/moment analyses that classical descriptions have provided, that are also typically limited to uniform and isotropic flows. Part of the difficulty can be traced to the fact that information offered by such analyses is not invertible. Given a process, its spectrum, for example, is specified. Knowledge of the spectrum, alone, yields only limited other information about the process.

Such turbulent-flow issues often pose questions regarding the geometrical properties of turbulence-generated fields. Examples of such issues include, heat and mass transfer in turbulent flows; mixing and chemically-reacting turbulent flows, requiring information about the surface-to-volume ratio of scalar level sets; aerooptics and optical-beam propagation through a turbulent medium, which (absent additional modeling and assumptions) require geometrical information about index-ofrefraction gradients; aeroacoustics and weak- and strong-wave propagation through turbulence, which rely on the geometrical properties of both scalar and velocity fields; and many others. While important progress has been made in these phenomena as well, which has derived considerable benefit from classical turbulence theory, in almost all cases, additional, often ad hoc, assumptions, variations, and models are employed, often implicitly.

More recently, the realization that Direct Numerical Simulation (DNS) methods cannot hope to represent turbulent phenomena at the high Reynolds numbers of interest, especially when coupled to other physical processes that must be computed in parallel, has led to the quest for sub-grid-scale (SGS) models that describe the behavior of scales smaller than those that can potentially be numerically resolved. Significantly, classical models do not yield the necessary SGS models, which also require additional structure and assumptions, as would be employed in Large Eddy Simulations (LES) calculations. Geometrical scaling information that would permit an extension of descriptions founded on an underresolved range of scales would facilitate this quest.

An important contribution that addressed some geometric-scaling issues was made by B. Mandelbrot (1975, 1982), who proposed that (power-law/self-similar) fractals could be used to describe level-set behavior of scalar and other turbulencegenerated fields. A considerable body of experimental and modeling work by many investigators followed these proposals, as discussed by Sreenivasan (1991, 1994). An extension of these proposals, necessitated by recent experiments and direct numerical simulations, will be discussed below. 


\section{Fractals and scale-dependent irregular level sets}

Mandelbrot $(1975,1977,1982)$, proposed to apply the notion of stochastic geometric self-similarity to describe a host of physical phenomena, including turbulence. Level sets in a $d$-dimensional embedding space conforming to this description he dubbed as fractal and can be covered by a number of elements (boxes, tiles, line segments), $N_{d}(\lambda)$, of size $\lambda$, given by,

$$
N_{d}(\lambda) \propto \lambda^{-D_{d}}
$$

where,

$$
D_{d}=-\frac{\mathrm{d} \log N_{d}(\lambda)}{\mathrm{d} \log \lambda},
$$

must be a constant. For level sets generated by turbulent flow, Eq. 1b must be regarded as potentially applicable over some finite range of scales, only, i.e.,

$$
\lambda_{\mathrm{i}} \equiv \max \left\{\lambda_{\nu}, \lambda_{\mathcal{D}}\right\} \ll \lambda_{\min }<\lambda<\lambda_{\max } \ll \delta .
$$

Here $\delta$ is the outer scale and $\lambda_{\mathrm{i}}$ is the inner scale, e.g., viscous-/diffusion-scale maximum, of the flow.

Irregular level sets need not be geometrically self-similar and may be characterized by a coverage, $N_{d}(\lambda)$, whose logarithmic derivative, $D_{d}(\lambda)$, may now be a function of scale, i.e.,

$$
D_{d}(\lambda)=-\frac{\mathrm{d} \log N_{d}(\lambda)}{\mathrm{d} \log \lambda}
$$

which we may dub the (scale-dependent) coverage dimension. Inverting Eq. 2a leads to,

$$
N_{d}(\lambda)=\exp \left\{\int_{\lambda}^{\delta} D_{d}\left(\lambda^{\prime}\right) \frac{\mathrm{d} \lambda^{\prime}}{\lambda^{\prime}}\right\},
$$

for a coverage count normalized at $\lambda=\delta$, i.e., for $N_{d}(\delta)=1$ (Takayasu 1982, 1992; Miller \& Dimotakis 1991; Dimotakis 1991; and Catrakis \& Dimotakis 1996a). A more complete discussion of these notions can be found in the review by Dimotakis \& Catrakis (1996).

Equations 2a,b are the counterparts of Eqs. 1b,a, that may be regarded as a special case, with the more relaxed definition of $D_{d}(\lambda)$ in the former now allowing their application over the whole range of flow scales. They will be used to analyze two-dimensional slices of the scalar field in the far-field of liquid-phase turbulent jets and of the density field in a Rayleigh-Taylor-instability flow. 


\section{Isoscalar geometry in turbulent jets}

In recent liquid-phase jet experiments (Catrakis \& Dimotakis 1996a), the fluid Schmidt number $\left(S c \simeq 2.0 \times 10^{3}\right)$ and flow Reynolds number $\left(R e \simeq 4.5 \times 10^{3}\right.$, $9.0 \times 10^{3}$, and $18 \times 10^{3}$ ) resulted in a jet-fluid concentration field with a complex, multiscale geometry. Laser-induced-fluorescence images of the scalar far field in this flow, in a plane normal to the jet axis, are shown in Fig. 1. They span the whole jet-fluid-concentration field, at the $z / d_{\mathrm{j}}=275$ downstream measurement location.
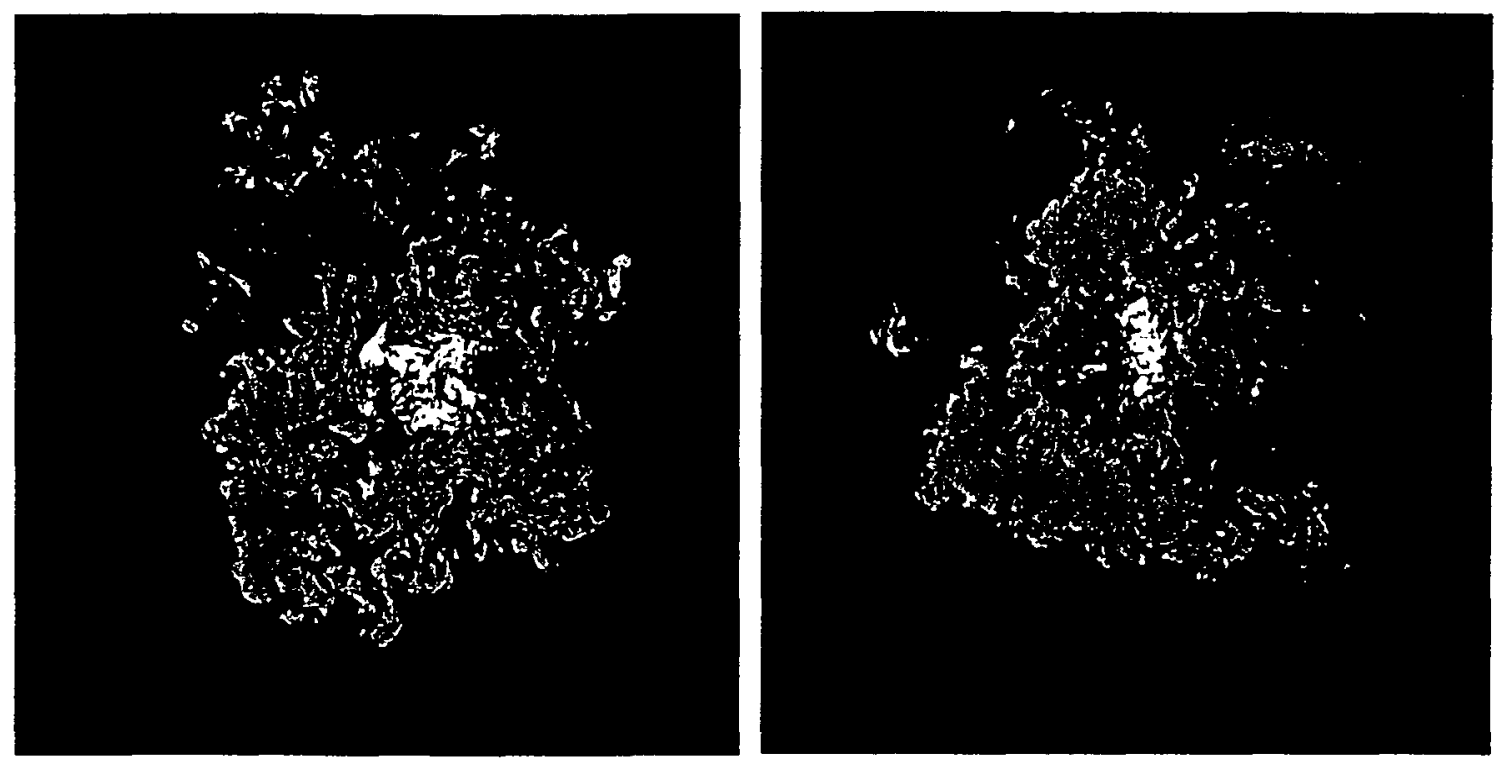

FIG. 1 Laser-induced fluorescence scalar-field data in a liquid-phase turbulent jet at $R e \simeq 4.5 \times 10^{3}$ (left) and $18 \times 10^{3}$ (right) in a plane normal to the jet axis in the far field $\left(z / d_{\mathrm{j}}=275\right)$. Color denotes jet-fluid concentration level.

Spatial spectra computed for the jet scalar-field data arc shown in Fig. 2. The 2-D spectrum (left) is for a single image realization $\left(R e=9 \times 10^{3}\right)$ and very-nearly axisymmetric. Radial spectra, obtained by azimuthal integration, are also shown (right) for the three Reynolds numbers investigated. As can be seen, other than axisymmetry, very little information is conveyed by the 2-D spectrum; similarly for the radially-averaged spectra, which, however, indicate a decreasing wavenumber content with increasing $R e$, in contrast to classically-expected behavior. The departure occurs at wavenumbers corresponding to scales roughly $1 / 3$ the image extent and are not the consequence of measurement resolution, which is adequate. 

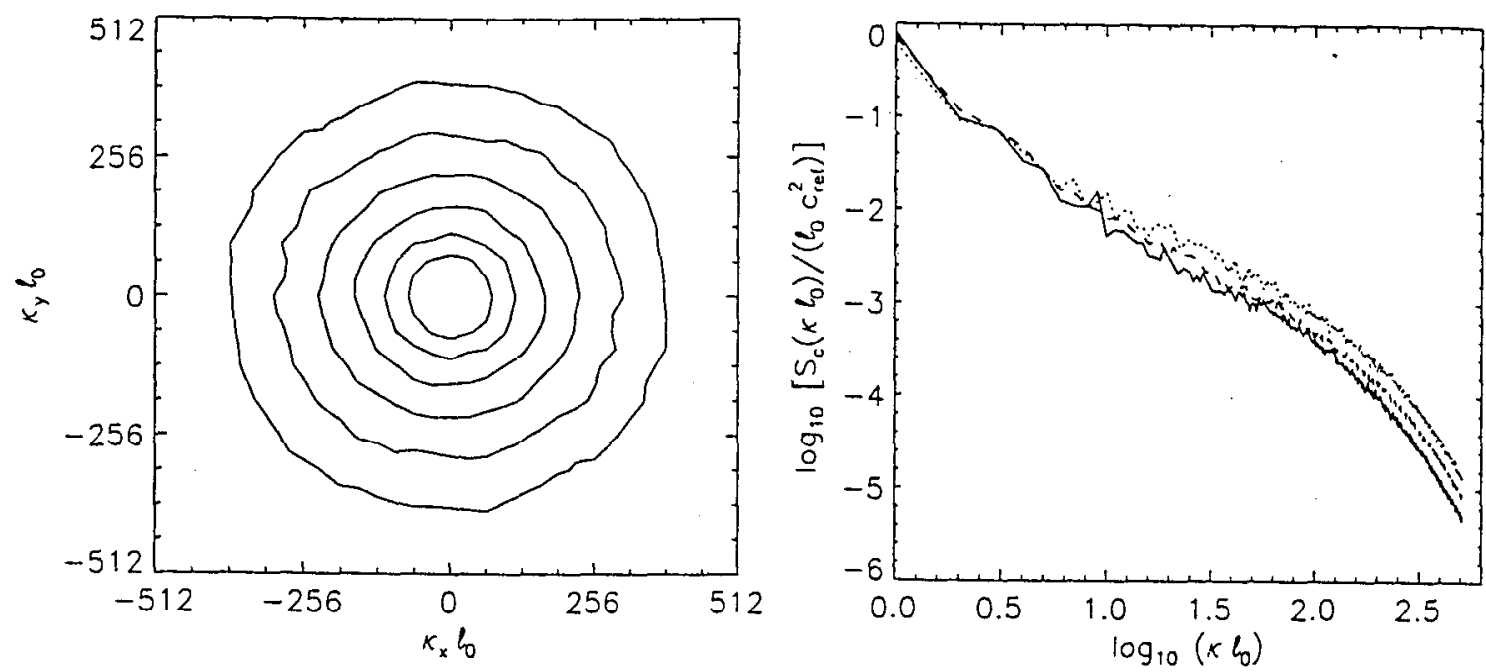

FIG. 2 Scalar spectrum for single image realization in a turbulent jet, at $R e=9 \times 10^{3}$ (left) and ensemble-averaged radial scalar spectra (right), for $R e=4.5 \times 10^{3}$, $9 \times 10^{3}$, and $18 \times 10^{3}$; lines of increasing solidity denote increasing $R e$.

The concentration data were thresholded at a level $c(x, y)=c_{2}$, corresponding to the peak in the scalar pdf at the two lower Reynolds numbers ( $c f$. Catrakis \& Dimotakis $1996 \mathrm{a}$, Fig. 8 ) and the $\delta_{\mathrm{b}}$-size bounding box was computed, i.e., the circumscribing rectangle of the level set. At this threshold, $\delta_{\mathrm{b}}$ is independent of $R e$, within measurement statistics. Figure 3 depicts selected stages of a typical coverage sequence of a $c_{2}$-level set, at $R e \simeq 9.0 \times 10^{3}$, through binary subdivisions of the bounding box. Size is here defined as the square-root of the box/tile area.

One $\delta_{\mathrm{b}}$-size tile covers the set, i.e., $N_{2}\left(\delta_{\mathrm{b}}\right)=1$, while four half-size tiles cover the set, i.e., $N_{2}\left(\lambda=\delta_{\mathrm{b}} / 2\right)=4$, yielding $D_{2}(\lambda) \rightarrow 2$, as $\lambda \rightarrow \delta_{\mathrm{b}}$. As $\lambda$ gets smaller, only a fraction of the total number of tiles is needed, i.e., $N_{2}(\lambda)<N_{2, \text { tot }}(\lambda)=$ $\left(\delta_{\mathrm{b}} / \lambda\right)^{2}$, and the coverage dimension decreases. Finally, at the smallest $\lambda$ 's, the coverage approximates the level-set contour. In this limit, $N_{2}(\lambda)$, increasing linearly with $\lambda$, as $\lambda \rightarrow 0$. The latter limit can be assessed by computing the normalized scalar level-set coverage length, $L(\lambda)$, from the coverage count, $N_{2}(\lambda)$, as a function of the coverage scale, $\lambda$, i.e.,

$$
\frac{L(\lambda)}{\delta_{\mathrm{b}}}=\frac{\lambda}{\delta_{\mathrm{b}}} N_{2}(\lambda) \rightarrow \text { const., as } \lambda \rightarrow 0 .
$$

This is plotted in Fig. 4 (left), for the three Reynolds numbers investigated. As can be seen, the data indicate a scale-independent level-set coverage length, at small scales; a limiting behavior attained for scales higher than the measurement resolution. The data also indicate a level-set coverage length that decreases with increasing $R e$. 

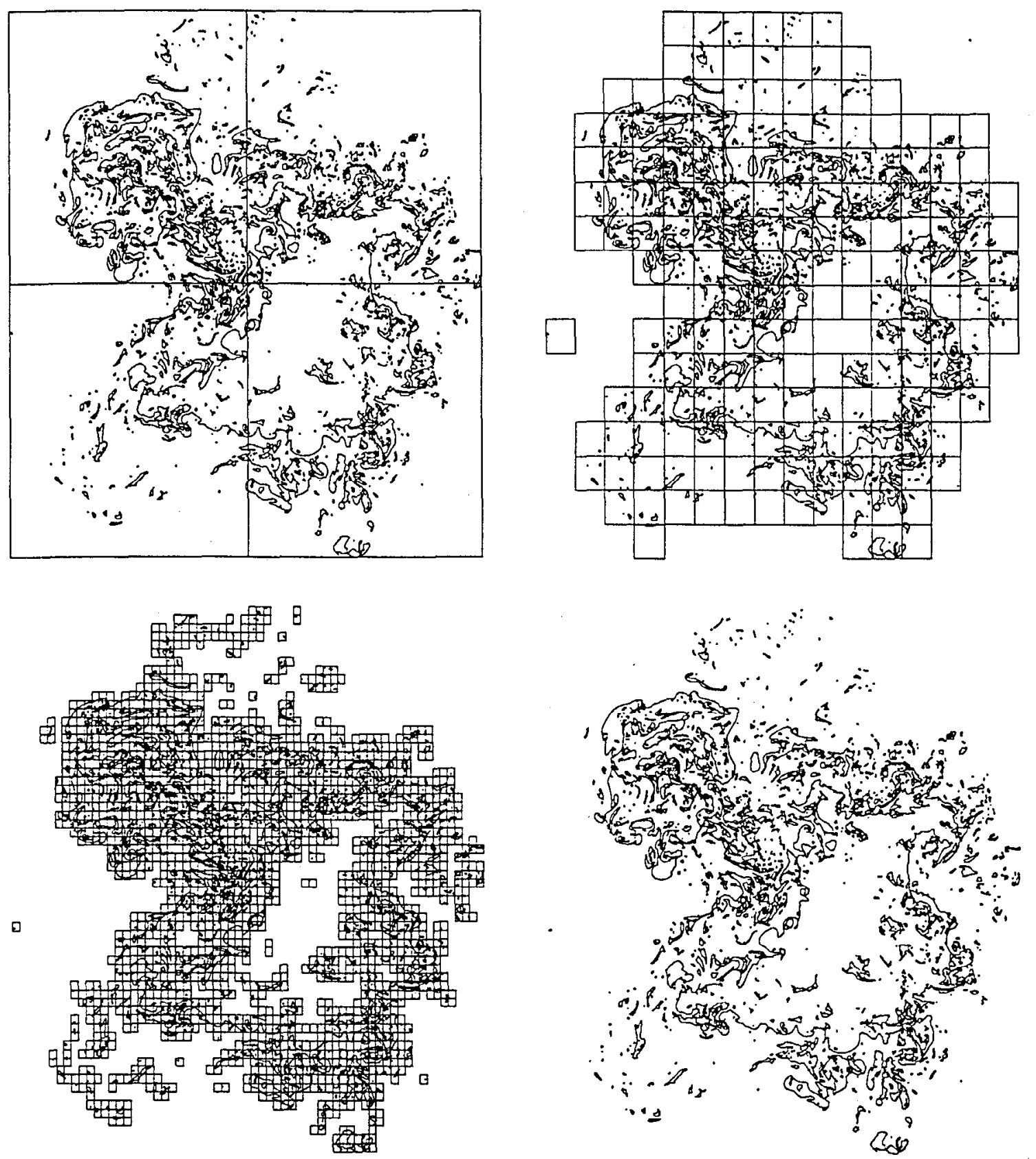

FIG. 3 Coverage sequence stages of a scalar level set in a liquid-phase turbulent jet $\left(R e \simeq 9.0 \times 10^{3}\right)$

The coverage dimension, $D_{2}(\lambda)$, is shown in Fig. 4 (right), for the Reynolds numbers investigated (Eq. 2a). It is a smoothly-increasing function of scale, bounded by its limiting value of unity (topological dimension), at the smallest scales, and 2 (embedding dimension), at the largest scales ( $c f$. , also, Miller \& Dimotakis 1991). A similar behavior obtains for lower and higher values of the scalar threshold. 

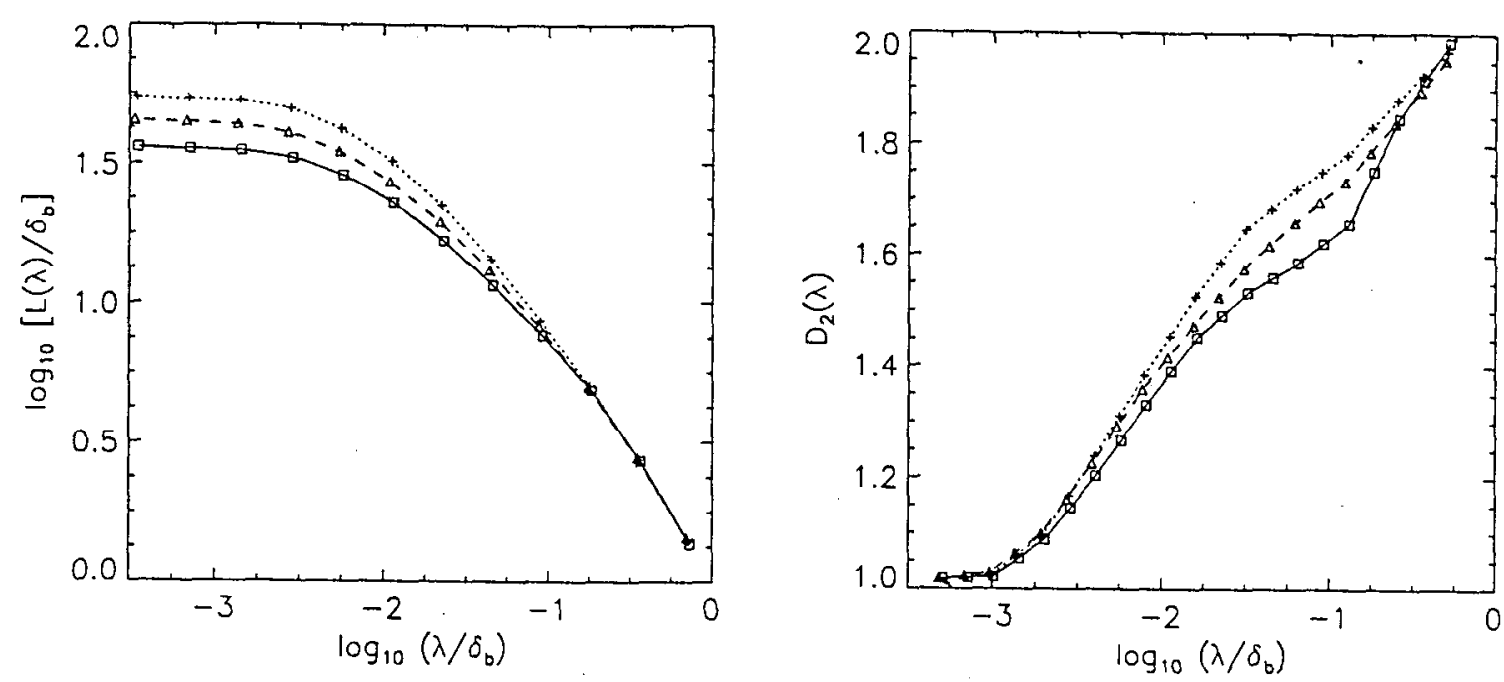

FIG. 4 Coverage length and coverage dimension as a function of (normalized) scale for scalar level sets in a turbulent jet. $R \mathrm{e} \simeq 4.5 \times 10^{3}$ : dotted/crosses; $R e \simeq 9.0 \times 10^{3}:$ dashed/triangles; $R e \simeq 18 \times 10^{3}:$ solid/squares .

Both the coverage length and dimension indicate less-convoluted level sets with increasing $R e$, in accord with the radially-averaged spatial-spectrum sequence (Fig. 2, right). The limiting value of $L(\lambda)$, as $\lambda \rightarrow 0$, and the coverage dimension, $D_{2}(\lambda)$, at medium-to-large scales, both decrease with incrcasing $R e$. These findings are consistent with enhanced mixing, relative to stirring, as Re increases, leading to improved local homogenization of the scalar field and geometrically-simpler scalar level sets. These, in turn, result in lower surface-to-volume ratios, with increasing Re. This is manifest in the comparison plot of sample $c_{2}$-level sets computed from $R e=4.5 \times 10^{3}$ (left) and $R e=18 \times 10^{3}$ (right) realizations (Fig. 5).

For such data, scalar level sets consist of individual (disjoint) "islands" and "lakes", depending on whether the interior is at a lower, or higher, scalar level, respectively. It is useful to analyze island/lake statistics, such as size and shape complexity. In the context of combustion, for example, an island would be associated with an unburnt fuel pocket in a non-premixed turbulent-jet flame. Such an analysis indicates that the size distribution of such features is well approximated by a log-normal pdf, at small-to-intermediate scales (Catrakis \& Dimotakis 1996b). Size here is defined as $\sqrt{A}$, with $A$ the individual island/lake area.

Returning to chemical reactions and combustion in non-premixed hydrocarbon turbulent flames, in which combustion is largely confined to the instantaneous stoichiometric (isoscalar) surface (Burke \& Schumann 1928), area-volume measures of 

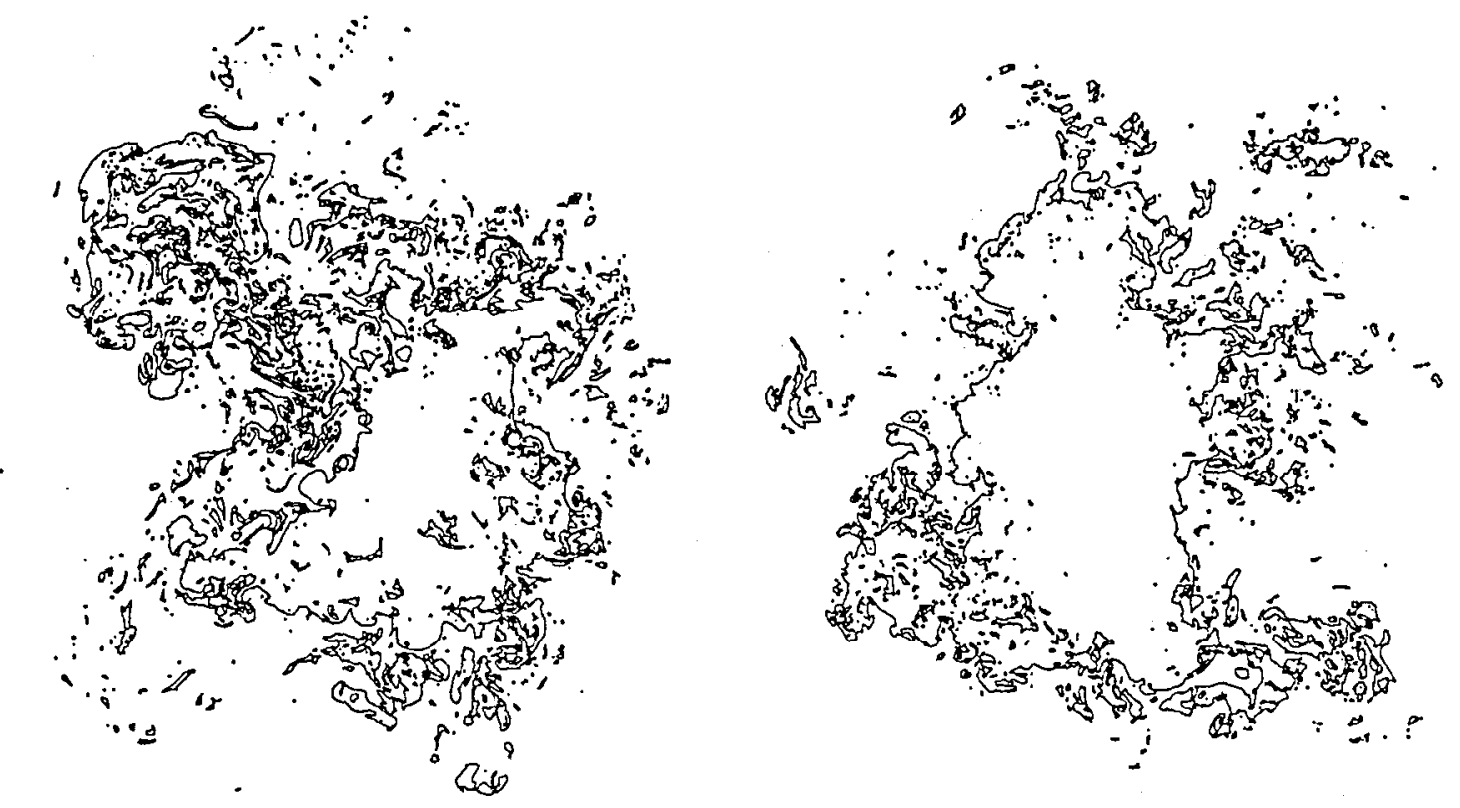

FIG. 5 Scalar $c_{2}$ level sets at $R e \simeq 4.5 \times 10^{3}$ (left) and $18 \times 10^{3}$ (right), indicating simpler topology at higher Reynolds number.

the isoscalar surface may be used to relate the local burning rate to the time required for the local consumption of unburnt fuel pockets. Such a measure, dubbed shape complexity, can be defined as,

$$
1 \leq \Omega_{2} \equiv \frac{P}{2(\pi A)^{1 / 2}} \leq \infty
$$

in 2-D, where $P$ is the perimeter and $A$ the area of an island or lake, with $\left(\Omega_{2}\right)_{\min }=1$ attained for a circle, and corresponding extensions for $\Omega_{d}$, for higher-dimensional embedding spaces. The liquid-phase jet data described above indicate that a power law over 3 decades in size ( 6 decades in area) provides a good approximation for the pdf of shape complexity. This behavior is equivalent to log-Poisson statistics for $\Omega_{2}$ (Catrakis \& Dimotakis 1998).

\section{Isoscalar geometry in Rayleigh-Taylor-instability flow}

A coverage analysis was also performed on isodensity data from a Navier-Stokes DNS study of the evolution of a Rayleigh-Taylor-instability flow, of a $S c \equiv \nu / \mathcal{D}=1$ fluid. The flow was initialized with a $\rho=3$ fluid on top and a $\rho=1$ fluid on the bottom, in a $256^{2} \times 512$ rectangular box. The three-dimensional DNS of the evolving flow was terminated when the spatial-resolution requirements could no longer be met by the fixed grid, at $R e_{\text {final }} \approx 1.1 \times 10^{3}$, based on the vertical extent and growth rate of the Rayleigh-Taylor mixing region (Cook 1998). 


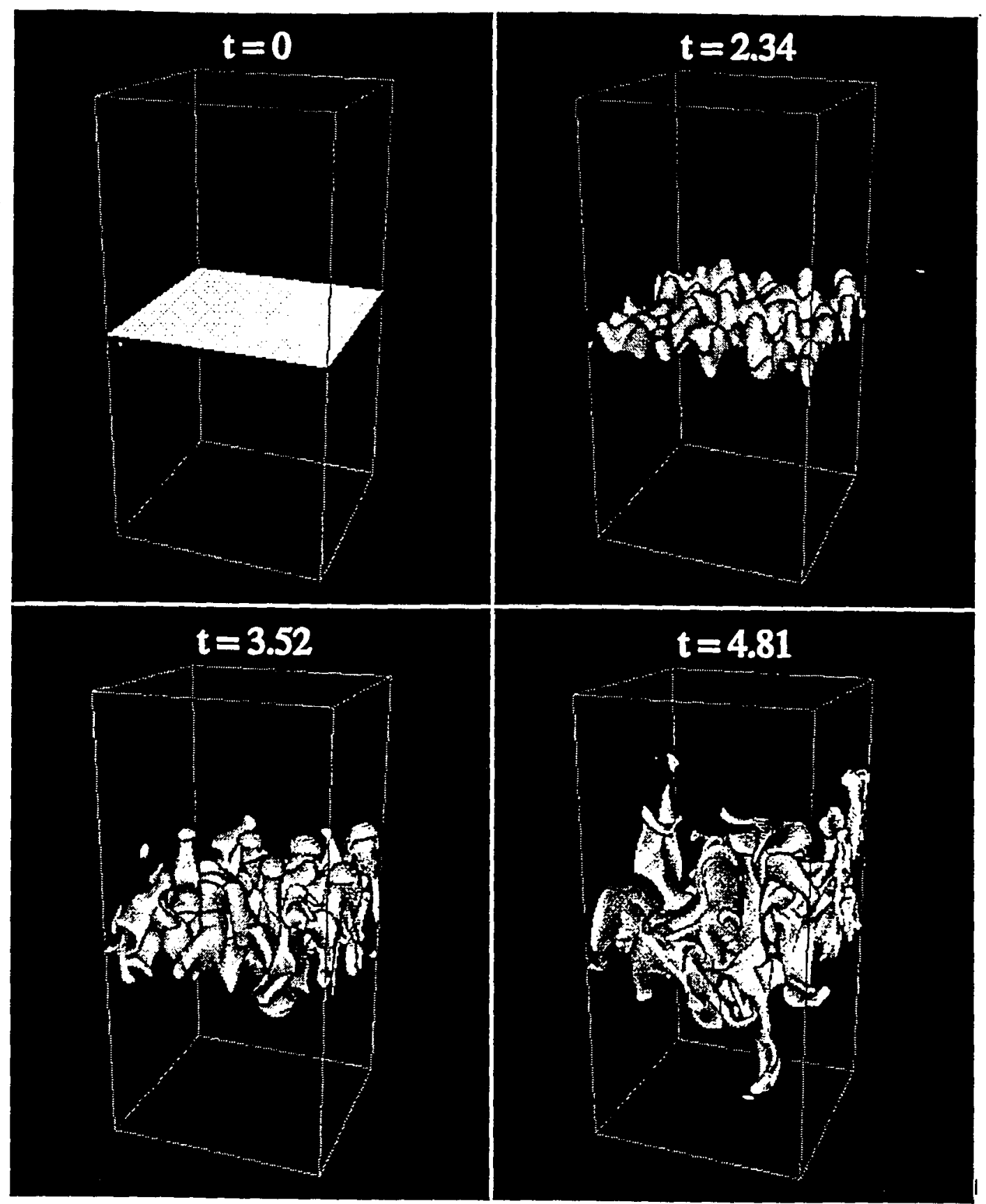

FrG.6 Rayleigh-Taylor-instability flow. $\rho=3$ fluid initially on top, $\rho=1$ fluid on the bottom. Plot of $\rho=2$ isosurface at indicated times.

The simulation utilized periodic boundary conditions in the boundary planes transverse to the acceleration vector, and no-slip at the top and bottom faces at the end of the long dimension of the box, which was aligned with the acceleration vector. A small-amplitude perturbation of the interface between the two fluids initialized the flow. Figure 6 illustrates the evolution of the $\rho=2$ isosurface. 

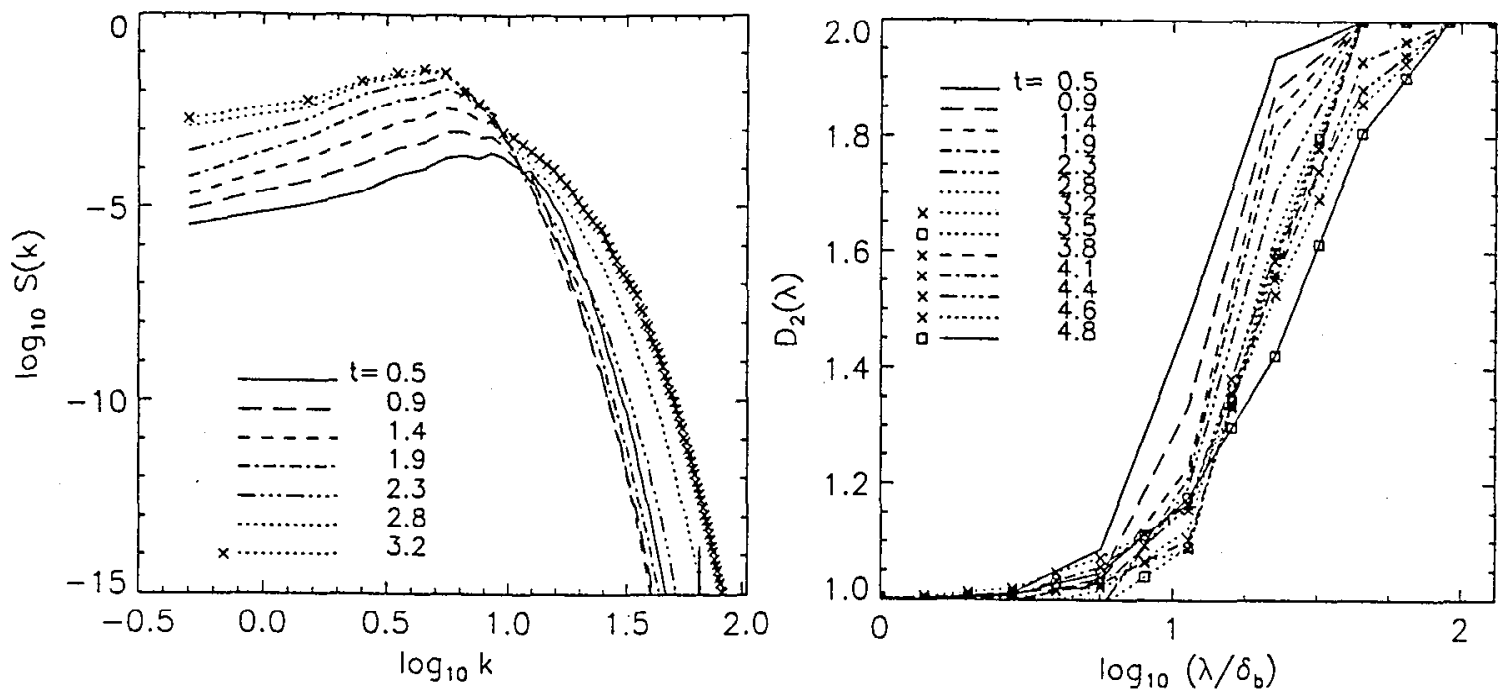

FIG. 7 Temporal evolution of scalar power spectrum (left) and coverage dimension (right) for a $2 \mathrm{D}$ slice (at mid-height) of the $\rho=2$ isoscalar surfacc in a numerical simulation of the Rayleigh-Taylor-instability flow.

Figure 7 (left) shows the time evolution of the spatial spectrum of the density field in the midheight plane, i.e., of $\rho(x, y, z=0)$, for the indicated progression in time. The spectrum initially exhibits a temporal progression to lower wavenumbers, a result of diffusive smoothing of the initial density-field pcrturbation (recall that $S c=1$ here). While the low wavenumber spectral content continues to increase, a sustained progression to higher wavenumbers with increasing time (for $t>1.4$ ), can be seen, plausibly as the Rayleigh-Taylor and other, secondary, flow instabilities take over, with the spectrum reflecting the growth of small-scale features at the midheight plane.

Figure 7 (right) plots the coverage dimension, $D_{2}(\lambda)$, for the $\rho(x, y, z=0)=2$ isodensity contours, in the same plane, computed by successive binary subdivisions of the midheight slice, as in the jet scalar-data analysis, above. The resulting scale-dependent coverage dimension $D_{2}(\lambda)$ can be seen to span the range of valucs from unity (the topological dimension), to 2 (the embedding dimension), smoothly transitioning between the two limiting values, at the smallest and the largest scales, respectively. Interestingly, the temporal progression indicated by $D_{2}(\lambda)$ is from small to large scales, for $t \lesssim 3.5$, i.e., opposite the high-wavenumber trend in the spectral analysis. It is not until near the end of the simulation (for $t \gtrsim 3.5$ ), where a reversal of this trend is exhibited, at small scales only. The reasons for this apparent disagreement are instructive and will be discussed below. 

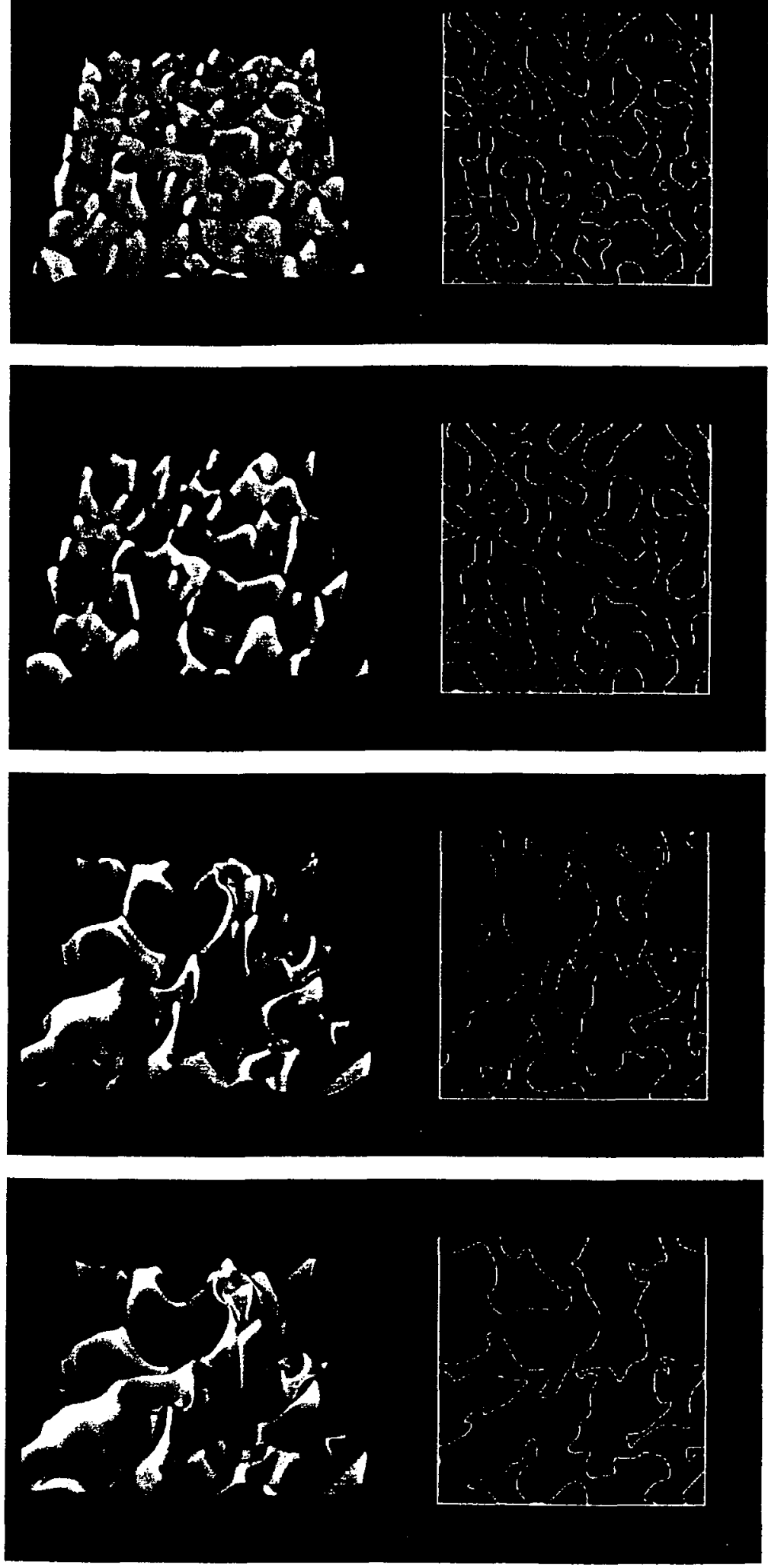

FIG. 8 Plot of $\rho(x, y, z=0 ; t)$ surfaces (left) and $\rho(x, y, z=0 ; t)=2$ contours (right), at times (top to bottom): $t=0.938,1.875,4.607$, and 4.810 . 
The spectrum measures the wavenumber content of the selected density field, $\rho(x, y, z=0 ; t)$, i.e., of the density surface over the midheight $(x, y)$-plane, while the level-set coverage analysis interrogates the geometry of the $\rho(x, y, z=0 ; t)=2$ contours in the midheight $(x, y)$-plane, i.e., the slice of the former, at the $\rho=2$ elevation. These data are depicted in Fig. 8, left and right, respectively, at the indicated times. As can be seen by comparing the two early-time ( $t=0.938$ and 1.875) top figures, the contours (right) are becoming smoother, while the density surface is developing sharp peaks. The latter are responsible for the spectral progression to higher wavenumbers. This trend continues for a while, with a subsequent transition that can be seen in the behavior of the density surfaces (left). These develop a more complicated topology, characterized by folds between local maxima and minima, at late times ( $c f$. Fig. 8, bottom: $t=4.607$ and 4.810). This also registers in the levelset contours that can now clearly be seen to develop small-scale features, on top of the larger-scale features that continue to increase in size.

Viewing the flow evolution through the three-dimensional density-field data (not discussed here) indicates that the likely cause of this transition is the development of secondary instabilities, of the Kelvin-Helmholtz type in the high-shear, near-midheight regions generated by the interpenetrating Rayleigh-Taylor fingers, and the formation of mushroom-like structures at their tips (cf. Fig. 6). The crossover in the coverage dimension, $D_{2}(\lambda)$, at small scales for late times revealed this transition, even though there is scant, if any, evidence for it in the spectral data. It is an attestation of the scale-local capability of the coverage analysis that such geometrical properties were clearly registered in those statistics. They were subsequently confirmed by computer-visualization of the corresponding field information.

\section{Conclusions}

Classical turbulence statistics and theories, while providing important guidance, do not explicitly address geometrical issues. For such issues, new tools are and will be required, such as the ones derived from the original self-similar fractal ideas, extended to accommodate the behavior of scale-dependent irregular level sets. Statistics that derive from the coverage analysis of level sets provide many useful geometric measures that complement information from classical theories and analysis of turbulence. Two turbulent-flow cases, involving experimental data in the far-field of liquid-phase turbulent jets and a low-Re realization of a $S c=1$ Rayleigh-Taylor-instability flow, confirm the need for and utility of such extensions. 
In particular, the scale-local nature of coverage analysis of irregular level sets makes it a better register of geometrical information, which is difficult to infer, at best, from spectral data alone.

\section{Acknowledgements}

This collaborative work was supported by the Air Force Office of Scientific Research, Grant Nos. F49620-94-1-0353 and F49620-98-1-0052, as part of an effort to study turbulent mixing; by Caltech's Center for Advanced Computing Research (CACR); and by the Academic Strategic Alliance Program from Lawrence Livermore National Laboratory (LLNL), under Department of Energy Contract W-7405-ENG-48. The DNS work on the Rayleigh-Taylor-instability flow was performed under the auspices of the Department of Energy, by Los Alamos National Laboratory (LANL), Contract W-7405-ENG-36, on the IBM ASCI-Pacific-Blue computer at Lawrence Livermore National Laboratory, as part of an effort to test LES models, while one of us (AC) was at LANL. We would also like to acknowledge discussions with Jerry Shan and Paul Miller, help by Paul Miller and Fokion Egolfopoulos with the text, as well as the expert assistance by Dan Lang on the digital-imaging, computing, and networking parts of the experimental effort on turbulent jets.

\section{References}

Batchelor, G. K. 1953 The Theory of Homogeneous Turbulence (Cambridge U.P., London).

Burke, S. P. \& Schumann, T. E. W. 1928 "Diffusion Flames," Ind. Eng. Chem. 20, 998.

Catrakis, H. J. \& Dimotakis, P. E. 1996a "Mixing in turbulent jets: scalar measures and isosurface geometry," J. Fluid Mech. 317, 369-406.

Catrakis, H. J. \& Dimotakis, P. E. 1996b "Scale Distributions and Fractal Dimensions in Turbulence," Phys. Rev. Lett. 77, 3795-3798.

Catrakis, H. J. \& Dimotakis, P. E. 1998 "Shape Complexity in Turbulence," Phys. Rev. Lett. 80, 968-971.

Cook, A. W. 1998 "Large-eddy simulation of variable-density flows," submitted to the J. Fluid Mech. . 
Dimotakis, P. E. 1991 "Fractals, dimensional analysis and similarity, and turbulence," Nonlinear Sci. Today \#2/91, pp. 1, 27-31.

Dimotakis, P. E. \& Catrakis, H. J. 1996 "Turbulence, fractals, and mixing," NATO Advanced Studies Institute series, Mixing: Chaos and Turbulence, GALCIT Report FM97-1.

FRISH, U. 1995 Turbulence. The Legacy of Kolmogorov (Cambridge U.P., Cambridge U.K.).

Hinze, J. O. 1975 Turbulence (2 ${ }^{\text {nd }}$ ed., McGraw-Hill).

Kolmogorov, A. N. 1941a "Interpolation and Extrapolation of Stationary Random Sequences," translated from the Russian by W. Doyle and I. Selin, RAND Corp. Memorandum RM-3090-PR (April 1962).

Kolmogorov, A. N. 1941b "On degeneration of isotropic turbulence in an incompresible fluid," C. R. Acad. Sci. U.R.S.S. 32, 538.

Kolmogorov, A. N. 1941c "Dissipation of energy in locally isotropic turbulence," Doklady Akad. Nauk. S.S.S.R. 66, 825.

MANDELBRot, B. B. 1975 "On the geometry of homogeneous turbulence, with stress on the fractal dimension of the iso-surfaces of scalars," J. Fluid Mech.72, 401-416.

Mandelbrot, B. B. 1977 Fractals. Form, Chance, and Dimension (W. H. Freeman \& Co., San Francisco).

Mandelbrot, B. B. 1982 The Fractal Geometry of Nature (W. H. Freeman \& Co., NY).

Miller, P. L. \& Dimotakis, P. E. 1991 "Stochastic geometric properties of scalar interfaces in turbulent jets," Phys. Fluids A 3, 168-177.

Monin, A. S. \& Yaglom, A. M. 1975 Statistical Fluid Mechanics: Mechanics of Turbulence II (Ed. J. Lumley, MIT Press, Cambridge, MA).

Sreenivasan, K. R. 1991 "Fractals and Multifractals in Fluid Turbulence," Ann. Rev. Fluid Mech. 23, 539-600.

Sreenivasan, K. R. 1994 "Fractals in Fluid Mechanics," Fractals 2, 253-263.

TAKaYASU, H. 1982 "Differential Fractal Dimension of Random Walk and Its Applications to Physical Systems," J. Phys. Soc. Japan 51, 3057-3064.

TAKaYasu, H. 1992 Fractals in the Physical Sciences (Wiley, Chichester, UK).

TAYLOR, G. I. 1938 "The spectrum of turbulence," Proc. Roy. Soc. A 164; 476. 